\title{
Zeros of some difference polynomials
}

\section{Shuangting Lan and Zongxuan Chen*}

\section{"Correspondence:} chzx@vip.sina.com

School of Mathematical Sciences, South China Normal University, Guangzhou, 510631, People's Republic of China

\begin{abstract}
In this paper, we study zeros of some difference polynomials in $f(z)$ and their shifts, where $f(z)$ is a finite order meromorphic function having deficient value $\infty$. These results improve previous findings.
\end{abstract}

\section{Introduction and results}

In this paper, we use the basic notions of Nevanlinna's theory (see [1]). In addition, we use the notations $\sigma(f)$ to denote the order of growth of the meromorphic function $f(z)$, and $\lambda(f)$ to denote the exponent of convergence of zeros of $f(z)$.

Hayman [2] studied the Picard values of a meromorphic function and their derivatives, and he obtained the following result.

Theorem A If $f(z)$ is a transcendental entire function, $n \geq 3$ is an integer, and a $(\neq 0)$ is a constant, then $f^{\prime}(z)-a f(z)^{n}$ assumes all finite values infinitely often.

Yang $[3,4]$ extended Theorem A to some differential polynomial in $f(z)$, where $f(z)$ is a transcendental meromorphic function satisfying $N(r, f)+N\left(r, \frac{1}{f}\right)=S(r, f)$.

Recently, many papers have focused on complex differences. They have obtained many new results on difference utilizing the value distribution theory of meromorphic functions. Some findings may be viewed as the corresponding difference analogues. First, recall some definitions. We call

$$
f(z)^{i_{\lambda, 0}} f\left(z+c_{1}\right)^{i_{\lambda, 1}} \ldots f\left(z+c_{k}\right)^{i_{\lambda, k}}
$$

is a monomial in $f(z)$ and its shifts $f\left(z+c_{1}\right), \ldots, f\left(z+c_{k}\right)$, where $c_{1}, \ldots, c_{k}$, are distinct nonzero complex constants, and $d(\lambda)=i_{\lambda, 0}+i_{\lambda, 1}+\cdots+i_{\lambda, k}$ is its degree. Further,

$$
P(z, f)=\sum_{\lambda \in I} a_{\lambda}(z) f(z)^{i_{\lambda, 0}} f\left(z+c_{1}\right)^{i_{\lambda, 1}} \cdots f\left(z+c_{k}\right)^{i_{\lambda, k}}
$$

is called a difference polynomial in $f(z)$ and its shifts, and $d(P)=\max _{\lambda \in I}\{d(\lambda)\}$ is its degree, where $I$ is a finite set of the index $\lambda=\left(i_{\lambda, 0}, \ldots, i_{\lambda, k}\right)$, and $a_{\lambda}(z)$ are meromorphic coefficients being small with respect to $f$ in the sense that $T\left(r, a_{\lambda}\right)=S(r, f), \lambda \in I$.

Zheng and Chen [5] obtained the following theorem, which may be considered as a difference counterpart of Theorem A.

(c) 2013 Lan and Chen; licensee Springer. This is an Open Access article distributed under the terms of the Creative Commons Attribution License (http://creativecommons.org/licenses/by/2.0), which permits unrestricted use, distribution, and reproduction in any medium, provided the original work is properly cited. 
Theorem B Let $f(z)$ be a transcendental entire function of finite order, and let $a, c$ be nonzero constants. Then, for any integer $n \geq 3, f(z+c)-a f(z)^{n}$ assumes all finite values $b \in \mathbb{C}$ infinitely often.

Chen [6] obtained the following Theorems C-E, which may be considered as another difference counterpart of Theorem A.

Theorem C Let $f(z)$ be a transcendental entire function offinite order, and let a, $c \in \mathbb{C} \backslash\{0\}$ be constants with $c$ such that $f(z+c) \not \equiv f(z)$. Set $\Psi_{n}(z)=\Delta f(z)-$ af $(z)^{n}$, where $\Delta f(z)=$ $f(z+c)-f(z)$ and $n \geq 3$ is an integer. Then $\Psi_{n}$ assumes all finite values infinitely often, and for every $b \in \mathbb{C}$ one has $\lambda\left(\Psi_{n}-b\right)=\sigma(f)$.

Theorem D Let $f(z)$ be a transcendental entire function of finite order with a Borel exceptional value 0 , and let $a, c \in \mathbb{C} \backslash\{0\}$ be constants, with $c$ such that $f(z+c) \not \equiv f(z)$. Then $\Psi_{2}(z)$ assumes all finite values infinitely often, and for every $b \in \mathbb{C}$ one has $\lambda\left(\Psi_{2}-b\right)=\sigma(f)$.

Zheng and Chen [7] extended the above Theorems B-D to a difference polynomial and proved the following theorem.

Theorem $\mathbf{E}$ Let $f(z)$ be a transcendental meromorphic function of finite order satisfying

$$
N(r, f)+N\left(r, \frac{1}{f}\right)=S(r, f) .
$$

Suppose that $P(z, f)$ is a difference polynomial of the form (1.1) and contains exactly one term of maximal total degree in $f(z)$ and its shifts. Then, for any given $a \in \mathbb{C} \backslash\{0\}$, the difference polynomial

$$
Q(z, f)=f(z)^{n}+P(z, f), \quad n \geq d(P)+2
$$

satisfies $\delta(a, Q(z, f))<1$. Therefore, $Q(z, f)$ takes every nonzero finite value infinitely often.

We observe that condition (1.2) can be weakened. It is well known that deficient value plays an important role in the theory of value distribution of meromorphic functions. In this paper, we consider the meromorphic function $f(z)$ having deficient value $\infty$ and obtain the following improvements of Theorem $\mathrm{E}$, which may be stated as follows.

Theorem 1.1 Let $f(z)$ be a transcendental meromorphic function of finite order, and let $P(z, f)(\not \equiv 0)$ be a difference polynomial of the form (1.1) with $k$ different shifts. Assume that $\delta(\infty, f)>1-\frac{1}{2 k+7}$. Then the difference polynomial

$$
Q(z, f)=f(z)^{n}+P(z, f), \quad n \geq d(P)+2,
$$

has infinitely many zeros and satisfies $\delta(0, Q(z, f))<1$.

If we add the condition $\bar{N}\left(r, \frac{1}{f}\right)=S(r, f)$, then the condition $\delta(\infty, f)>1-\frac{1}{2 k+7}$ in Theorem 1.1 can be weakened as $\delta(\infty, f)>1-\frac{1}{2 k+5}$, and we obtain the following theorem. 
Theorem 1.2 Let $f(z)$ be a transcendental meromorphic function of finite order, and let $P(z, f)(\not \equiv 0)$ be a difference polynomial of the form (1.1) with $k$ different shifts. Assume that $\delta(\infty, f)>1-\frac{1}{2 k+5}$ and $\bar{N}\left(r, \frac{1}{f}\right)=S(r, f)$, then the difference polynomial

$$
Q(z, f)=f(z)^{n}+P(z, f), \quad n \geq d(P)+1
$$

has infinitely many zeros and satisfies $\delta(0, Q(z, f))<1$.

Example 1.1 For $f(z)=e^{z}+1, c=\log 3$, we have $\Psi_{2}(z)=-f(z)^{2}+\Delta f(z)=-e^{2 z}-1$. Here $\Psi_{2}(z) \neq-1$, which shows that the condition $\bar{N}\left(r, \frac{1}{f}\right)=S(r, f)$ in Theorem 1.2 cannot be replaced with $\bar{N}\left(r, \frac{1}{f-c}\right)=S(r, f)$, where $c$ is a nonzero constant.

If we add the additional condition $\bar{N}(r, f)=S(r, f)$, then we obtain the following.

Theorem 1.3 Let $f(z)$ be a transcendental meromorphic function of finite order satisfying

$$
\delta(\infty, f)>\frac{2}{3}, \quad \bar{N}(r, f)=S(r, f) .
$$

Then the difference polynomial $Q(z, f)$ defined by (1.3) has infinitely many zeros and satisfies $\delta(0, Q(z, f))<1$.

Theorem 1.4 Let $f(z)$ be a transcendental meromorphic function of finite order with $\delta(\infty, f)>0$ satisfying

$$
\bar{N}(r, f)+\bar{N}\left(r, \frac{1}{f}\right)=S(r, f)
$$

Then the difference polynomial $Q(z, f)$ defined by (1.4) has infinitely many zeros and satisfies $\delta(0, Q(z, f))<1$.

Remark 1.1 For any given $b \in \mathbb{C}$, applying Theorems 1.1-1.4 to the difference polynomial $Q^{*}(z, f)=Q(z, f)-b$, then we have $\delta(b, Q(z, f))<1$.

Remark 1.2 If $Q(z, f)=a_{n}(z) f(z)^{n}+P(z, f)$, where $a_{n}(z)$ is small with respect to $f$, applying Theorems 1.1-1.4 to the difference polynomial $Q^{*}(z, f)=\frac{Q(z, f)}{a_{n}(z)}$, then we also have $\delta(0, Q(z, f))<1$.

Applying Theorems 1.1-1.4 to the difference polynomial $\Psi_{n}=\Delta f(z)-a f(z)^{n}$, we get the following Corollaries 1.5-1.8, which extend Theorems C, D.

Corollary 1.5 Let $f(z)$ be a transcendental meromorphic function of finite order with $\delta(\infty, f)>\frac{8}{9}$, and let $a, c \in \mathbb{C} \backslash\{0\}$ be constants with $c$ such that $\Delta f(z)$ does not reduce to any constant and $n \geq 3$ is an integer. Then, for every $b \in \mathbb{C}$, one has $\delta\left(b, \Psi_{n}\right)<1$.

Corollary 1.6 Let $f(z)$ be a transcendental meromorphic function of finite order satisfying

$$
\delta(\infty, f)>\frac{2}{3}, \quad \bar{N}(r, f)=S(r, f),
$$


and let $a, c \in \mathbb{C} \backslash\{0\}$ be constants, with $c$ such that $\Delta f(z)$ does not reduce to any constant and $n \geq 3$ is an integer. Then, for every $b \in \mathbb{C}$, one has $\delta\left(b, \Psi_{n}\right)<1$.

Corollary 1.7 Let $f(z)$ be a transcendental meromorphic function offinite order satisfying

$$
\delta(\infty, f)>\frac{6}{7}, \quad \bar{N}\left(r, \frac{1}{f}\right)=S(r, f),
$$

and let $a, c \in \mathbb{C} \backslash\{0\}$ be constants, with c such that $\Delta f(z)$ does not reduce to any constant. Then, for every $b \in \mathbb{C}$, one has $\delta\left(b, \Psi_{2}\right)<1$.

Corollary 1.8 Let $f(z)$ be a transcendental meromorphic function of finite order satisfying $\delta(\infty, f)>0$ and

$$
\bar{N}(r, f)+\bar{N}\left(r, \frac{1}{f}\right)=S(r, f)
$$

and let $a, c \in \mathbb{C} \backslash\{0\}$ be constants with $c$ such that $\Delta f(z)$ does not reduce to any constant. Then, for every $b \in \mathbb{C}$, one has $\delta\left(b, \Psi_{2}\right)<1$.

\section{Lemmas for the proof of the theorem}

Lemma 2.1 [8] Given two distinct complex constants $\eta_{1}, \eta_{2}$, letf be a meromorphic function of finite order. Then we have

$$
m\left(r, \frac{f\left(z+\eta_{1}\right)}{f\left(z+\eta_{2}\right)}\right)=S(r, f) .
$$

Lemma 2.2 [9] Let $f(z)$ be a nonconstant meromorphic function of finite order, then for any given constant $c(\neq 0)$, we have

$$
N(r, f(z+c))=N(r, f)+S(r, f), \quad \bar{N}(r, f(z+c))=\bar{N}(r, f)+S(r, f)
$$

possibly outside of an exceptional set of finite logarithmic measure.

Lemma 2.3 [4] Let $f(z)$ be a transcendental meromorphic function, and let $P(f)$ be an algebraic polynomial in $f$ of the form

$$
P(f)=a_{n}(z) f(z)^{n}+a_{n-1}(z) f(z)^{n-1}+\cdots+a_{1}(z) f(z)+a_{0}(z)
$$

where $a_{n}(z) \not \equiv 0, a_{j}(j=0, \ldots, n)$ are small with respect to $f$. Then

$$
T(r, P(f))=n T(r, f)+S(r, f) \text { as } r \rightarrow \infty
$$

Using the same method as in the proof of Lemma 2.3, we show the following.

Lemma 2.4 Let $f(z)$ be a transcendental meromorphic function with $\delta(\infty, f)>0$, and let $P(f)$ be an algebraic polynomial in $f$ of the form $(2.1)$, where $a_{n}(z) \neq 0, a_{j}(z)$ satisfy $m\left(r, a_{j}\right)=$ $S(r, f), j=0, \ldots, n$, then

$$
m(r, P(f)) \leq n m(r, f)+S(r, f)
$$


Proof Since $a_{j}(z)$ satisfy $m\left(r, a_{j}\right)=S(r, f), j=0, \ldots, n$, then

$$
\begin{aligned}
m(r, P(f)) & \leq m\left(r, f\left(a_{n} f^{n-1}+a_{n-1} f^{n-2}+\cdots+a_{2} f+a_{1}\right)\right)+m\left(r, a_{0}\right)+O(1) \\
& \leq m(r, f)+m\left(r,\left(a_{n} f^{n-1}+a_{n-1} f^{n-2}+\cdots+a_{2} f+a_{1}\right)\right)+m\left(r, a_{0}\right)+O(1) \\
& \leq m(r, f)+m\left(r, f\left(a_{n} f^{n-2}+a_{n-1} f^{n-3}+\cdots+a_{2}\right)\right)+m\left(r, a_{1}\right)+m\left(r, a_{0}\right)+O(1) \\
& \leq 2 m(r, f)+m\left(r, f\left(a_{n} f^{n-3}+a_{n-1} f^{n-4}+\cdots+a_{3}\right)\right)+\sum_{j=0}^{2} m\left(r, a_{j}\right)+O(1) \\
& \ldots \\
& \leq n m(r, f)+\sum_{j=0}^{n} m\left(r, a_{j}\right)+O(1) \\
& =n m(r, f)+S(r, f) .
\end{aligned}
$$

Thus,

$$
m(r, P(f)) \leq n m(r, f)+S(r, f)
$$

Lemma 2.5 [10] Let $f$ be a transcendental meromorphic solution of finite order of a difference equation of the form

$$
U(z, f) P(z, f)=Q(z, f)
$$

where $U(z, f), P(z, f)$ and $Q(z, f)$ are difference polynomials such that the total degree $\operatorname{deg}_{f} U(z, f)=n$ in $f(z)$ and its shifts, and $\operatorname{deg}_{f} Q(z, f) \leq n$. Moreover, we assume that all coefficients $a_{\lambda}(z)$ in (2.2) are small in the sense that $T\left(r, a_{\lambda}\right)=S(r, f)$ and that $U(z, f)$ contains just one term of maximal total degree in $f(z)$ and its shifts. Then, for each $\varepsilon>0$, we have

$$
m(r, P(z, f))=S(r, f)
$$

possibly outside of an exceptional set of finite logarithmic measure.

Yang and Laine in [11] further pointed out the following.

Remark 2.1 If $P(z, f)$ and $Q(z, f)$ are differential-difference polynomials in $f(z)$ and its shifts with coefficients $a_{\lambda}(z)$ satisfy $m\left(r, a_{\lambda}\right)=S(r, f)$, then Lemma 2.5 still holds.

\section{Proof of Theorems 1.1-1.4}

Proof of Theorem 1.1 We assert that $Q(z, f)$ does not reduce to any constant. In fact if $Q(z, f) \equiv C_{1}$, where $C_{1}$ is some constant, then

$$
f(z)^{n}=-P(z, f)+C_{1} .
$$


Applying Lemma 2.5 to (3.1), since $n \geq d(P)+2$, we have

$$
m(r, f)=S(r, f) .
$$

Since $\delta(\infty, f)=\delta>0$, then for any given $\varepsilon_{0}\left(0<\varepsilon_{0}<\delta\right)$ and sufficiently large $r$, we have

$$
m(r, f) \geq\left(\delta-\varepsilon_{0}\right) T(r, f) .
$$

By (3.2) and (3.3), we get

$$
T(r, f)=S(r, f),
$$

which is impossible. So, $Q(z, f)$ does not reduce to any constant.

Next we rearrange the expression difference polynomial $Q(z, f)$ by collecting together all terms having the same total degree and then writing $Q(z, f)$ in the form

$$
Q(z, f)=f(z)^{n}+\sum_{j=0}^{d(P)} \widetilde{a}_{j}(z) f(z)^{j},
$$

where the coefficients $\tilde{a}_{j}(z), j=0,1, \ldots, d(P)$ are the sum of finitely many terms of the type

$$
a_{\lambda}(z) \prod_{j=1}^{\tau_{\lambda}}\left(\frac{f\left(z+c_{j}\right)}{f(z)}\right)^{i_{\lambda, j}} .
$$

By Lemma 2.1 and our assumption concerning the coefficients $a_{\lambda}$, we obtain

$$
m\left(r, \tilde{a}_{j}\right)=S(r, f), \quad j=0,1, \ldots, d(P) .
$$

By (3.4), (3.5) and Lemma 2.4, we have

$$
m(r, Q(z, f)) \leq n m(r, f)+S(r, f) .
$$

By Lemma 2.2, we have

$$
\begin{aligned}
N(r, Q(z, f)) & \leq n N(r, f(z))+d(P) \sum_{j=1}^{k} N\left(r, f\left(z+c_{j}\right)\right)+S(r, f) \\
& =(n+k d(P)) N(r, f)+S(r, f)
\end{aligned}
$$

and

$$
\begin{aligned}
\bar{N}(r, Q(z, f)) & \leq \bar{N}(r, f(z))+\sum_{j=1}^{k} \bar{N}\left(r, f\left(z+c_{j}\right)\right)+O(1) \\
& \leq(k+1) \bar{N}(r, f)+S(r, f) .
\end{aligned}
$$


Thus,

$$
\begin{aligned}
T(r, Q(z, f)) & =m(r, Q(z, f))+N(r, Q(z, f)) \\
& \leq(n+k d(P)) T(r, f)+S(r, f) .
\end{aligned}
$$

So, $S(r, Q(z, f)) \leq S(r, f)$.

Differentiating both sides of (1.3), we obtain that

$$
Q^{\prime}(z, f)=n f^{n-1} f^{\prime}+P^{\prime}(z, f)
$$

Multiplying both sides of (1.3) by $\frac{Q^{\prime}}{Q}$ and subtracting from (3.8), we get

$$
f^{n-1}\left(n f^{\prime}-\frac{Q^{\prime}}{Q} f\right)=\frac{Q^{\prime}}{Q} P(z, f)-P^{\prime}(z, f)
$$

or

$$
f^{n-2} f\left(n f^{\prime}-\frac{Q^{\prime}}{Q} f\right)=\frac{Q^{\prime}}{Q} P(z, f)-P^{\prime}(z, f) .
$$

Now assert $n f^{\prime}-\frac{Q^{\prime}}{Q} f \not \equiv 0$. Otherwise $\frac{Q^{\prime}}{Q}=n \frac{f^{\prime}}{f}$, i.e., $Q=C_{2} f^{n}$ for a suitable constant $C_{2}$ leading to

$$
\left(C_{2}-1\right) f(z)^{n}=P(z, f) .
$$

We can see that $C_{2} \neq 1$, for $P(z, f) \not \equiv 0$. Since $n>d(P)$ by assumption and Lemma 2.5 , (3.11) implies $m(r, f)=S(r, f)$, which shows that $T(r, f)=S(r, f)$, again by (3.3). It is a contradiction.

Applying Lemma 2.5 and Remark 2.1 to (3.9) and (3.10) respectively, again by assumption $n \geq d(P)+2$, we obtain

$$
m\left(r, n f^{\prime}-\frac{Q^{\prime}}{Q} f\right)=S(r, f)
$$

and

$$
m\left(r, f\left(n f^{\prime}-\frac{Q^{\prime}}{Q} f\right)\right)=S(r, f) .
$$

By (3.6), we get that

$$
\begin{aligned}
N\left(r, n f^{\prime}-\frac{Q^{\prime}}{Q} f\right) & \leq N(r, f)+\bar{N}(r, f)+\bar{N}(r, Q)+\bar{N}\left(r, \frac{1}{Q}\right)+O(1) \\
& \leq N(r, f)+(k+2) \bar{N}(r, f)+\bar{N}\left(r, \frac{1}{Q}\right)+S(r, f)
\end{aligned}
$$

and

$$
N\left(r, f\left(n f^{\prime}-\frac{Q^{\prime}}{Q} f\right)\right) \leq 2 N(r, f)+\bar{N}(r, f)+\bar{N}(r, Q)+\bar{N}\left(r, \frac{1}{Q}\right)+O(1)
$$




$$
\leq 2 N(r, f)+(k+2) \bar{N}(r, f)+\bar{N}\left(r, \frac{1}{Q}\right)+S(r, f) .
$$

By (3.12)-(3.15), we obtain

$$
\begin{aligned}
T\left(r, n f^{\prime}-\frac{Q^{\prime}}{Q} f\right) & =m\left(r, n f^{\prime}-\frac{Q^{\prime}}{Q} f\right)+N\left(r, n f^{\prime}-\frac{Q^{\prime}}{Q} f\right) \\
& \leq N(r, f)+(k+2) \bar{N}(r, f)+\bar{N}\left(r, \frac{1}{Q}\right)+S(r, f)
\end{aligned}
$$

and

$$
\begin{aligned}
T\left(r, f\left(n f^{\prime}-\frac{Q^{\prime}}{Q} f\right)\right) & =m\left(r, f\left(n f^{\prime}-\frac{Q^{\prime}}{Q} f\right)\right)+N\left(r, f\left(n f^{\prime}-\frac{Q^{\prime}}{Q} f\right)\right) \\
& \leq 2 N(r, f)+(k+2) \bar{N}(r, f)+\bar{N}\left(r, \frac{1}{Q}\right)+S(r, f) .
\end{aligned}
$$

Therefore,

$$
\begin{aligned}
T(r, f) & \leq T\left(r, n f^{\prime}-\frac{Q^{\prime}}{Q} f\right)+T\left(r, f\left(n f^{\prime}-\frac{Q^{\prime}}{Q} f\right)\right)+O(1) \\
& \leq 3 N(r, f)+2(k+2) \bar{N}(r, f)+2 \bar{N}\left(r, \frac{1}{Q}\right)+S(r, f),
\end{aligned}
$$

or

$$
T(r, f) \leq(2 k+7) N(r, f)+2 \bar{N}\left(r, \frac{1}{Q}\right)+S(r, f) .
$$

For any given $\varepsilon\left(0<\varepsilon<\delta-1+\frac{1}{2 k+7}\right)$, we have

$$
T(r, f) \leq(2 k+7)(1-\delta+\varepsilon) T(r, f)+2 \bar{N}\left(r, \frac{1}{Q}\right)+S(r, f),
$$

or

$$
(1-(2 k+7)(1-\delta+\varepsilon)+o(1)) T(r, f) \leq 2 \bar{N}\left(r, \frac{1}{Q}\right)
$$

By (3.7) and (3.19), we have

$$
(1-(2 k+7)(1-\delta+\varepsilon)+o(1)) T(r, Q) \leq 2(n+k d(P)) \bar{N}\left(r, \frac{1}{Q}\right)
$$

which leads to

$$
\varlimsup_{r \rightarrow \infty} \frac{\bar{N}\left(r, \frac{1}{Q}\right)}{T(r, Q)} \geq \frac{1-(2 k+7)(1-\delta+\varepsilon)}{2(n+k d(P))}>0 .
$$

Therefore,

$$
\delta(0, Q)=1-\varlimsup_{r \rightarrow \infty} \frac{N\left(r, \frac{1}{Q}\right)}{T(r, Q)}<1 .
$$


Proof of Theorem 1.3 Using the same method as in the proof of Theorem 1.1, we see that (3.1)-(3.18) hold. By assumption $\bar{N}(r, f)=S(r, f)$ and (3.16)-(3.18), we have

$$
\begin{aligned}
T(r, f) & \leq 3 N(r, f)+2(k+2) \bar{N}(r, f)+2 \bar{N}\left(r, \frac{1}{Q}\right)+S(r, f), \\
& =3 N(r, f)+2 \bar{N}\left(r, \frac{1}{Q}\right)+S(r, f) .
\end{aligned}
$$

For any given $\varepsilon, 0<\varepsilon<\delta-\frac{2}{3}$, where $\delta=\delta(\infty, f)>\frac{2}{3}$, we have

$$
(1-3(1-\delta+\varepsilon)+o(1)) T(r, f) \leq 2 \bar{N}\left(r, \frac{1}{Q}\right)
$$

By (3.7), we have

$$
(1-3(1-\delta+\varepsilon)+o(1)) T(r, Q) \leq 2(n+k d(P)) \bar{N}\left(r, \frac{1}{Q}\right)
$$

which leads to

$$
\varlimsup_{r \rightarrow \infty} \frac{\bar{N}\left(r, \frac{1}{Q}\right)}{T(r, Q)} \geq \frac{1-3(1-\delta+\varepsilon)}{2(n+k d(P))}>0 .
$$

Therefore,

$$
\delta(0, Q)=1-\varlimsup_{r \rightarrow \infty} \frac{N\left(r, \frac{1}{Q}\right)}{T(r, Q)}<1 .
$$

Proof of Theorem 1.2 The proof is similar to the proof of Theorem 1.1.

We also obtain (3.1)-(3.9), (3.12), (3.14) and (3.16), by assumption that $n \geq d(P)+1$.

By logarithmic derivative lemma, we get

$$
m\left(r, n \frac{f^{\prime}}{f}-\frac{Q^{\prime}}{Q}\right) \leq S(r, f)+S(r, Q)=S(r, f) .
$$

Since $\bar{N}\left(r, \frac{1}{f}\right)=S(r, f)$, by (3.6), we get

$$
\begin{aligned}
N\left(r, n \frac{f^{\prime}}{f}-\frac{Q^{\prime}}{Q}\right) & \leq \bar{N}(r, f)+\bar{N}\left(r, \frac{1}{f}\right)+\bar{N}(r, Q)+\bar{N}\left(r, \frac{1}{Q}\right)+O(1) \\
& \leq(k+2) \bar{N}(r, f)+\bar{N}\left(r, \frac{1}{Q}\right)+S(r, f)
\end{aligned}
$$

By (3.20) and (3.21), we have

$$
\begin{aligned}
T\left(r, n \frac{f^{\prime}}{f}-\frac{Q^{\prime}}{Q}\right) & =m\left(r, n \frac{f^{\prime}}{f}-\frac{Q^{\prime}}{Q}\right)+N\left(r, n \frac{f^{\prime}}{f}-\frac{Q^{\prime}}{Q}\right) \\
& \leq(k+2) \bar{N}(r, f)+\bar{N}\left(r, \frac{1}{Q}\right)+S(r, f) .
\end{aligned}
$$


Then,

$$
\begin{aligned}
T(r, f) & \leq T\left(r, n f^{\prime}-\frac{Q^{\prime}}{Q} f\right)+T\left(r, n \frac{f^{\prime}}{f}-\frac{Q^{\prime}}{Q}\right)+O(1) \\
& \leq N(r, f)+2(k+2) \bar{N}(r, f)+2 \bar{N}\left(r, \frac{1}{Q}\right)+S(r, f),
\end{aligned}
$$

or

$$
T(r, f) \leq(2 k+5) N(r, f)+2 \bar{N}\left(r, \frac{1}{Q}\right)+S(r, f)
$$

For any given $\varepsilon, 0<\varepsilon<\delta-1+\frac{1}{2 k+5}$, where $\delta=\delta(\infty, f)>1-\frac{1}{2 k+5}$, by (3.7), we obtain

$$
(1-(2 k+5)(1-\delta+\varepsilon)) T(r, Q) \leq 2(n+k d(P)) \bar{N}\left(r, \frac{1}{Q}\right),
$$

which leads to

$$
\varlimsup_{r \rightarrow \infty} \frac{\bar{N}\left(r, \frac{1}{Q}\right)}{T(r, Q)} \geq \frac{1-(2 k+5)(1-\delta+\varepsilon)}{2(n+k d(P))}>0 .
$$

Therefore,

$$
\delta(0, Q)=1-\varlimsup_{r \rightarrow \infty} \frac{N\left(r, \frac{1}{Q}\right)}{T(r, Q)}<1 .
$$

Proof of Theorem 1.4 Using the same method as in the proof of Theorem 1.2, we obtain (3.23). By (1.5) and (3.23), we have

$$
T(r, f) \leq N(r, f)+2 \bar{N}\left(r, \frac{1}{Q}\right)+S(r, f) .
$$

For any given $\varepsilon, 0<\varepsilon<\delta$, where $\delta=\delta(\infty, f)$, we have

$$
(\delta-\varepsilon+o(1)) T(r, f) \leq 2 \bar{N}\left(r, \frac{1}{Q}\right) .
$$

Then by (3.7), we have

$$
(\delta-\varepsilon+o(1)) T(r, Q) \leq 2(n+k d(P)) \bar{N}\left(r, \frac{1}{Q}\right),
$$

which leads to

$$
\varlimsup_{r \rightarrow \infty} \frac{\bar{N}\left(r, \frac{1}{Q}\right)}{T(r, Q)} \geq \frac{\delta-\varepsilon}{2(n+k d(P))}>0 .
$$

Therefore,

$$
\delta(0, Q)=1-\varlimsup_{r \rightarrow \infty} \frac{N\left(r, \frac{1}{Q}\right)}{T(r, Q)}<1 .
$$




\section{Competing interests}

The authors declare that they have no competing interests.

\section{Authors' contributions}

All authors drafted the manuscript, read and approved the final manuscript.

\section{Acknowledgements}

The authors would like to thank the editor and the referees for their constructive comments to improve the readability of our paper. The project was supported by the National Natural Science Foundation of China $(11171119,11126145)$.

\section{Received: 25 March 2013 Accepted: 14 June 2013 Published: 1 July 2013}

\section{References}

1. Yang, L: Value Distribution Theory and Its New Research. Science Press, Beijing (1982) (in Chinese)

2. Hayman, WK: Picard values of meromorphic functions and their derivatives. Ann. Math. 70(1), 9-42 (1959)

3. Yang, CC: On deficiencies of differential polynomials. Math. Z. 116, 197-204 (1970)

4. Yang, CC: On deficiencies of differential polynomials II. Math. Z. 125, 107-112 (1972)

5. Zheng, XM, Chen, ZX: On the value distribution of some difference polynomials. J. Math. Anal. Appl. 397, 814-821 (2013)

6. Chen, ZX: On value distribution of difference polynomials of meromorphic functions. Abstr. Appl. Anal. 2011, Article ID 239853 (2011)

7. Zheng, XM, Chen, ZX: On deficiencies of some difference polynomials. Acta Math. Sin. 54, 983-992 (2011) (in Chinese)

8. Halburd, RG, Korhonen, RJ: Difference analogue of the lemma on the logarithmic derivative with applications to difference equations. J. Math. Anal. Appl. 314, 477-487 (2006)

9. Chiang, YM, Feng, SJ: On the Nevanlinna characteristic of $f(z+\eta)$ and difference equations in the complex plane. Ramanujan J. 16, 105-129 (2008)

10. Laine, I, Yang, CC: Clunie theorems for difference and q-difference polynomials. J. Lond. Math. Soc. 2(76), 556-566 (2007)

11. Yang, CC, Laine, I: On analogies between nonlinear difference and differential equations. Proc. Jpn. Acad., Ser. A, Math Sci. 86(1), 10-14 (2010)

doi:10.1186/1687-1847-2013-194

Cite this article as: Lan and Chen: Zeros of some difference polynomials. Advances in Difference Equations 2013 2013:194.

\section{Submit your manuscript to a SpringerOpen ${ }^{\circ}$ journal and benefit from:}

- Convenient online submission

Rigorous peer review

- Immediate publication on acceptance

- Open access: articles freely available online

- High visibility within the field

- Retaining the copyright to your article 